\title{
Parfum de bonté et odeur de sainteté
}

Les enjeux de l'olfaction dans l'éducation religieuse des jeunes filles au $\mathrm{XIX}^{\mathrm{e}}$ siècle

\section{Érika Wicky}

\section{(2) OpenEdition}

\section{Journals}

Édition électronique

URL : http://journals.openedition.org/aes/1853

DOI : 10.4000/aes.1853

ISSN : 2258-093X

Éditeur

Laboratoire LISAA

Référence électronique

Érika Wicky, "Parfum de bonté et odeur de sainteté », Arts et Savoirs [En ligne], 11 | 2019, mis en ligne le 15 juillet 2019, consulté le 06 septembre 2019. URL : http://journals.openedition.org/aes/1853 DOI : 10.4000/aes.1853

Ce document a été généré automatiquement le 6 septembre 2019

Centre de recherche LISAA (Littératures SAvoirs et Arts) 


\title{
Parfum de bonté et odeur de sainteté
}

\author{
Les enjeux de l'olfaction dans l'éducation religieuse des jeunes filles au \\ XIX ${ }^{\mathrm{e}}$ siècle
}

Érika Wicky

Sur quels pieds tombez-vous, parfums de Madeleine?

Musset, Rolla, $1833^{1}$

1 Émanations invisibles et impalpables, les odeurs corporelles demeurent un mystère pour les sciences jusqu'au début $\mathrm{du} \mathrm{Xx}^{\mathrm{e}}$ siècle $^{2}$. Qu'elles soient envisagées comme ondes ou comme molécules, elles sont liées, dans le discours médical et religieux, à des considérations morales en vertu d'une corrélation étroite entre senteurs et vertus ${ }^{3}$. Associée au corps et à la sexualité, l'odeur est souvent invoquée pour jeter l'opprobre sur les prostituées ${ }^{4}$ ou bien elle marque le passage à l'âge adulte, l'entrée dans la condition de femme, d'amante ou d'épouse ${ }^{5}$. Aussi les prescriptions concernant la maitrise des odeurs corporelles et l'usage des parfums jouent-elles un rôle majeur dans l'éducation des jeunes filles, notamment dans la seconde moitié $d u \mathrm{XIX}^{\mathrm{e}}$ siècle où l'éducation religieuse connaît un nouvel essor ${ }^{6}$. Elles s'inscrivent dans les stratégies de contrôle mises en place par les instances médicales, sociales ou religieuses lesquelles insistent sur la double nécessité de respirer peu d'odeurs fortes et de réduire ses propres émanations olfactives, qu'elles soient ou non agréables.

2 Toutefois, en dépit d'une exigence commune de réguler le corps et les odeurs, les différents conseils adressés aux jeunes filles dans les guides de piété et de convenance ou dans la presse féminine et les réclames de parfumeurs sont parfois contradictoires. Ainsi, les normes d'hygiène varient considérablement entre le discours religieux et le discours médical qui partagent, néanmoins, des conceptions morales similaires. De même, les usages contribuent à rendre les discours équivoques: on ne saurait, par exemple, encourager une hygiène rigoureuse tout en réprouvant l'utilisation de fragrances à une époque où l'emploi répandu de savons parfumés constitue la norme ${ }^{7}$. Ces nombreuses contradictions trouvent une illustration dans la célèbre formule de George Sand : « Nous élevons [les jeunes filles] tant que nous pouvons comme des saintes, puis nous les livrons 
comme des pouliches $»^{8}$. En effet, avant même de devenir épouses, les jeunes filles doivent à la fois plaire puisqu'elles sont destinées au mariage, tout en répondant aux exigences morales d'une éducation religieuse qui les voue à devenir un relais de l'Église au sein de la famille ${ }^{9}$. Les séductions de la vertu sont suffisamment célébrées à l'époque pour que cette double exigence de l'éducation des jeunes filles ne paraisse pas paradoxale au premier abord. Cependant, les pratiques d'hygiène préconisées pour plaire à Dieu et celles destinées à plaire aux hommes sont très différentes, de sorte que se cristallisent autour de la question des odeurs corporelles des positions très contrastées en matière d'éducation physique et morale des jeunes filles.

Il s'agira donc de se pencher sur les recommandations adressées aux jeunes filles issues de la bourgeoisie en ce qui concerne leur odeur personnelle et d'explorer parallèlement les usages pédagogique, moraux et religieux des métaphores olfactives. Les tensions et les contradictions autour de la question des odeurs et de leur régulation n'opposent pas seulement les prescriptions médicales aux préceptes religieux et aux réclames des parfumeurs. Elles se font jour au sein même de la rhétorique et des pratiques religieuses.

\section{Hygiène et éducation religieuse}

L'éducation catholique donnée aux jeunes filles de la seconde moitié du xix siècle s'attache moins à instruire qu'à former de bonnes ménagères et de pieuses mères de famille ${ }^{10}$. Aussi insiste-t-elle sur la nécessité d'être soignée. Le logement doit être à la fois propre et rangé à la manière de la cellule de sœur Hélène que découvre George Sand, encore couventine : «Je fus agréablement surprise de la trouver d'une propreté exquise et toute parfumée de l'odeur du jasmin qui montait du préau jusqu'à sa fenêtre. La pauvre sœur était propre aussi ; elle avait sa robe de serge violette neuve; ses petits objets de toilette bien rangés sur une table attestaient le soin qu'elle prenait de sa personne ${ }^{11}$. L'hygiène corporelle fait également l'objet de prescriptions strictes : « Ne portez jamais ni linge ni vêtement malpropres ou déchirés ; soignez vos cheveux ; lavez-vous tous les jours la figure et plusieurs fois par jour les mains s'il est nécessaire. Que votre chaussure soit toujours en bon état $»^{12}$. L'ordonnancement des conseils dispensés par cette religieuse qui encourage les jeunes filles à se soucier essentiellement de la propreté de leur habillement et de ce qui en dépasse, signale l'ambiguïté du terme « toilette » qui incluait alors tout ce qui relevait de la parure, c'est-à-dire le vêtement, la coiffure et les soins de propreté. Plus loin, la religieuse limite les évocations de la propreté à celle de la blancheur du linge, sans mentionner une seule fois le bain qui était pourtant prôné alors par les hygiénistes, comme en témoigne la floraison de bains publics à cette époque ${ }^{13}$.

5 Ce décalage entre les règles imposées aux couventines et les normes en vigueur en matière d'hygiène suscite beaucoup de suspicion, notamment de la part de Théophile Gautier qui demande à sa fille, élevée au couvent: «Est-ce qu'on te lave au moins?» Cette dernière, Judith Gautier, poursuit le récit de la visite paternelle par ces mots :

Ce bain que mon père avait exigé pour moi et auquel on avait consenti, par crainte de perdre une élève, n'allait pas sans causer un grand embarras. C'était un événement insolite, pour lequel rien n'était disposé, et qui inspirait une sourde réprobation: le premier degré, peut-être, des pompes de Satan... On avait des hochements de tête, des haussements d'épaule, des yeux levés vers le ciel, et la sœur Dodo me confiait, innocemment, que le bain de la religieuse consistait, tout simplement, à secouer sa chemise $!^{14}$ 
6 Le caractère exceptionnel de ce bain, pris en chemise, illustre la nature des pratiques d'hygiène répandues dans les couvents et en laisse imaginer les conséquences olfactives. Elles soulèvent de vives critiques de la part des médecins. Si Jean-Baptiste Venot, par exemple, loue en 1857 le dévouement des religieuses qui accueillent les prostituées syphilitiques dans leurs maisons de convalescence, il n'en déplore pas moins les conséquences sanitaires de la prohibition du bain :

Certes, les efforts moraux tentés dans cet établissement par les dignes Sœurs qui le desservent méritent les plus grands éloges ; mais les soins de santé, de salubrité et de convalescence leur sont radicalement inconnus: elles imputent à péché la moindre ablution, le plus innocent lavage; le bain est une superfluité mondaine dont le nom n'est jamais prononcé dans le couvent. Étonnez-vous donc des récidives après cela. ${ }^{15}$

7 Deux décennies plus tard, le Docteur Auguste Galopin, à la fois plus radical et plus explicite dans ses formulations, reprend à son compte l'argument moral, pour épingler cette fois les dérives auxquelles peuvent mener les pratiques en usage dans les couvents. En effet, la «négligence de l'eau », provoquant prurit et démangeaisons, habitue les jeunes filles à se gratter et les familiarise avec de nouvelles sensations, les entraînant sur la pente de l'onanisme. Il conclut son argumentation par une condamnation sans appel : «Les pensions et les couvents de jeunes filles, où les religieuses défendent de se laver par là sous peine de pécher devraient être supprimées (sic) sans pitié : c'est une calamité morale publique. $»^{16}$

De même, l'enfermement des couventines est redouté des médecins qui préconisent plutôt le grand air et l'exercice. L'hygiéniste Adrien Coriveaud dresse ainsi un tableau accablant des effets délétères de la promiscuité dans un espace non aéré :

Et ce n'est pas tout; de l'haleine la plus fraîche et la plus pure de la plus idéale des vierges s'échappent, en nombre immense, des particules organiques, des miasmes... oui, oui, je sais fort bien que cela n'est pas galant, mais cela est vrai et il faut que vous le sachiez. Enfermez dans une chambre dont vous aurez pris soin de boucher jusqu'aux moindres fissures, vingt des plus belles, des plus ravissantes jeunes filles que vous pourrez trouver, de celles dont la grâce vaporeuses et les charmes divins inspirent aux poètes leurs strophes les plus harmonieuses, et laissez-les causer entre elles, chanter, danser, vivre en un mot leur vie ordinaire pendant seulement vingt-quatre heures, puis pénétrez dans ce petit gynécée. L'enivrant parfum que dès le début avaient dégagé autour d'elles ces vingt jeunes personnes, est maintenant remplacé par une odeur d'une horrible fétidité ; de ces vingt bouches mignonnes, de ces vingt poitrines qui peuvent être de décevants modèles de statues virginales se sont exhalés des flots de vapeur d'eau chargés de tous les déchets organiques que produit incessamment la vie en activité. ${ }^{17}$

9 En révélant une corporalité odorante, le confinement dépouille les jeunes filles du halo de pureté fantasmée dont elles sont d'ordinaire entourées.

Si les pratiques d'hygiène préconisées par les institutions religieuses et par les médecins diffèrent considérablement, elles n'en poursuivent pas moins un objectif commun, celui de préserver les bonnes mœurs en réprimant les plaisirs sensuels. Pour cette raison, le discours religieux et le discours médical s'accordent à condamner l'usage des parfums qui relèvent de la coquetterie et stimulent les sens. Il est donc recommandé aux jeunes filles de ne pas en porter, mais aussi de s'en tenir éloignées, voire de bannir les fleurs odoriférantes de leur chambre à coucher ${ }^{18}$. Ces craintes et ces mises en garde ne tardent pas à essaimer dans les romans qui contribuent à les répandre auprès d'un public plus large. Ainsi, dans La Faute de l'abbé Mouret, Zola met en scène le suicide d'Albine, asphyxiée 
par les effluves des fleurs étalées dans sa chambre ${ }^{19}$. S'appuyant également sur les conceptions médicales de son temps, Edmond de Goncourt retrace la passion sensuelle et mortifère d'une jeune fille, Chérie, pour les senteurs que lui procurent les parfumeurs du Second Empire ${ }^{20}$. Jean-Baptiste Fonssagrives, quant à lui, se montre attentif, en sa qualité d'hygiéniste, aux manœuvres de ces derniers lesquels, à l'instar de César Birotteau, se réclament de la science pour vanter et vendre leurs produits. L'hygiène, à ses yeux, ne saurait servir de caution aux stratégies commerciales des parfumeurs :

Depuis longtemps, la parfumerie fait les yeux doux à l'hygiène; [...] elle a senti le besoin d'abriter ses bonnes intentions sous un nom vénéré, et les parfums hygiéniques, les cosmétiques hygiéniques, les essences hygiéniques, s'échappent à rangs pressés de la cornue des parfumeurs. L'hygiène n'a rien à voir à tout cela ; elle suspecte toujours la sincérité de l'étiquette, et elle voudrait que ces engins d'une coquetterie aux abois et ces stimulants d'une sensibilité maladive restassent dans un certain monde qui, n'ayant rien à voir au parfum des bonnes mœurs et à l'odeur d'une réputation irréprochable, remplace ces aromes par ceux, plus faciles à se procurer, qui sortent de l'officine des parfumeurs. ${ }^{21}$

11 Alors que l'usage des parfums a longtemps été considéré comme suspect parce qu'il pouvait être destiné à masquer un manque d'hygiène ${ }^{22}$, il est envisagé ici comme un signe de mauvaises mœurs, corollaire, dans le registre moral, de la malpropreté. Aux accents médicaux et moralisateurs du propos de Fonssagrives se mêlent également des considérations sociales puisqu'il présente le parfum comme un marqueur de classe, différenciant notamment les femmes issues de la bourgeoisie des demi-mondaines ${ }^{23}$. Ainsi, alors que le discours médical et le discours religieux cherchent à établir une nette démarcation entre propreté et coquetterie (même s'ils ne la délimitent pas de la même manière), les parfumeurs s'ingénient à brouiller cette distinction en proposant des produits ambigus (savons parfumés, eaux de toilette, etc.) qu'ils font largement connaître par la publicité et qui deviennent des objets en vogue.

\section{Métaphores olfactives}

Une menace d'opprobre sociale est perceptible dans chacune des recommandations adressées aux jeunes filles en matière d'olfaction de sorte que leurs pratiques se trouvent gouvernées par la crainte de sentir mauvais, d'être trop parfumées ou bien de n'être pas à la mode. Cependant, face aux plaisirs et aux séductions déployés par les étalages des parfumeurs, qui se démocratisent de plus en plus, le propos pédagogique, hygiéniste ou religieux adressé aux jeunes filles offre lui aussi une "gamme » de parfums, envisagés, cette fois, sur le mode métaphorique : au sacrifice des parfums terrestres encouragé par l'imagerie dédiée à Marie-Madeleine (fig. 1) ${ }^{24}$ se substituent ainsi le " parfum des bonnes mœurs» ou "l'odeur d'une réputation irréprochable» évoqués plus haut par Fonssagrives. De sorte que Marie Oberthal, dans ses conseils à une jeune fille, formule ce souhait: «Oh! ma chère enfant, puisse une semblable émotion te ramener aux saintes joies de la vie du foyer recueillie et vertueuse, toute parfumée de chastes amours, toute remplie de suaves souvenirs. $»^{25}$ 


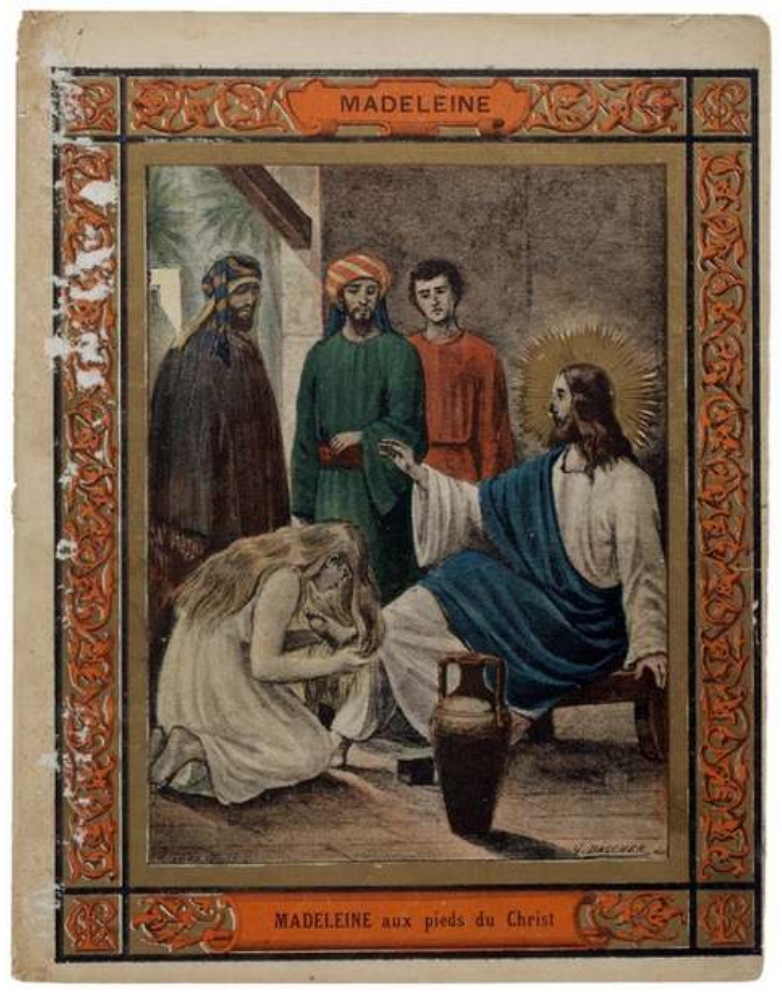

Fig. 1

Georges Dascher, Madeleine - Les noms de nos Filles, couverture de cahier, vers 1892, 22x17 cm Musée national de l'éducation.

La métaphore associant bonne odeur et vertu est certes motivée par le rapport de cause à effet que l'on concevait alors entre la propreté et les bonnes mœurs ${ }^{26}$, mais elle l'est aussi par la dimension immatérielle du parfum. En effet, le parfum, comme l'esprit, dans l'acception chimique du terme, est " comparé au souffle » en raison de sa "subtilité » ${ }^{27}$. Ce statut intermédiaire, que la science ne sait pas encore expliquer, fait du parfum un comparant privilégié pour donner une forme sensible à des notions abstraites. L'innocence, la pudeur, la chasteté sont ainsi autant de parfums de jeunes filles qu'il convient de préserver ${ }^{28}$. La référence récurrente à l'olfaction vient s'inscrire en contrepoint des textes dénigrant les plaisirs sensuels et incitant les jeunes filles à réduire le plus possible les stimulations sensorielles ${ }^{29}$. Elle constitue un argument efficace pour combattre le matérialisme dans les deux acceptions du terme puisqu'elle offre aux jeunes filles des images pour penser le rapport entre l'âme et le corps, voire la transsubstantiation, tout en les incitant à réduire leur usage de produits de luxe ${ }^{30}$.

La comparaison entre les vertus que la jeune fille est encouragée à cultiver et le parfum s'exprime le plus souvent à travers la métaphore de la femme-fleur, la fleur se référant ici principalement à la fleur des champs. Dépourvue d'animalité et de sensualité propre, celle-ci incarne, en effet, un idéal de pudeur, de modestie et de simplicité donné en exemple aux jeunes filles ${ }^{31}$. Marc Monnier exploite ce parallèle entre la fleur odoriférante et la jeune fille vertueuse dans un poème construit en deux parties, la première faisant l'éloge d'une fleur appelée Beauté et la seconde lui préférant un parfum nommé Bonté.

Mais il est un parfum céleste

Plus fécond encore en douceur,

Qui d'une fleur simple et modeste 
Peut faire une divine fleur.

Arome du lis angélique,

Baume de la rose mystique

Par la terre au ciel emprunté,

Comme l'encens des sacrifices,

Il répand de saintes délices...

- Et ce parfum, c'est la bonté !32

Les jeunes filles sont encouragées à s'engager sur la voie de la bonté pour goûter des plaisirs supérieurs à ceux qu'offrent les sens : « Les sensualistes ne savent pas les joies de la chasteté, le parfum que répand la rose blanche et le bonheur de la perfection ", observe ainsi l'historien et journaliste Jean-Baptiste Capefigue ${ }^{33}$. Véritable lieu commun, la métaphore du parfum floral préside également aux mises en garde contre la disparition de l'innocence, prompte, comme les senteurs, à s'évaporer : "Garde toi bien, ma Flavie, d'abuser du droit d'intéresser et de plaire », observe Jean-Nicolas Bouilly dans Conseils à ma fille; « La fleur printanière, exposée sans cesse aux rayons du soleil, perd son parfum avant l'été, se fane et tombe décolorée ; tandis que celle qui fleurit lentement à l'abri d'un modeste feuillage conserve longtemps sa fraîcheur, et charme encore ceux qui la découvrent dans l'arrière-saison $\aleph^{34}$. Dans le même ordre d'idées, l'évocation de la fugacité du parfum floral devient un lieu commun de la littérature d'édification afin d'inciter les jeunes filles, que l'on souhaite garder au foyer, à fuir les mondanités ${ }^{35}$. Mais la référence à l'olfaction a bien d'autres ressorts; elle vise également à souligner les propriétés spécifiques du parfum en opérant une distinction entre extraits de fleurs et parfums composés ou en faisant allusion à la mémoire olfactive ${ }^{36}$.

\section{Ivresses de l'encens}

L'éducation religieuse prescrit ainsi aux jeunes filles de rechercher des parfums abstraits qui évoquent métaphoriquement les vertus dont on voudrait les voir parées. Ce faisant, elle répond à la sensibilité de la seconde moitié $\mathrm{du}$ xIx $\mathrm{x}^{\mathrm{e}}$ siècle durant laquelle l'industrie de la parfumerie connaît des développements scientifiques et techniques majeurs accompagnés d'un important succès commercial ${ }^{37}$. Mais elle s'inscrit aussi dans une tradition plus ancienne, puisque les textes religieux font souvent de l'odeur agréable un signe d'élection divine, telle l'odeur de sainteté, élément récurrent dans les récits hagiographiques ${ }^{38}$. Cette odeur de sainteté prend une dimension exemplaire dans l'éducation des jeunes filles qui doivent rechercher, plus modestement, une « odeur de bonté ».

17 Toutefois, la sublimation du corps à travers le parfum fait également l'objet de contestations, notamment à la fin du siècle. En effet, à partir des années 1880 , suite au recul du poids politique de la religion catholique, elle suscite des critiques d'autant plus nombreuses qu'elles peuvent s'exprimer librement. L'anticléricalisme ambiant et le positivisme de l'époque créent un climat propice à l'interprétation des phénomènes religieux en termes médicaux. Telle est ainsi l'explication de l'odeur de sainteté proposée par Augustin Galopin, à l'appui des recherches d'un professeur new yorkais :

L'odeur de sainteté n'est pas, d'après Hammond, une simple figure de rhétorique; c'est l'expression d'une sainte névrose parfumant la peau d'effluves plus ou moins agréables, au moment du paroxysme religieux extatique; absolument semblable à l'expression de la névrose amoureuse parfumant également, et par les mêmes lois psycho-physiologiques, la peau satinée d'une femme adorée, que vous respirez à 
plein cerveau et qui n'est pas résignée du tout, comme la trop sainte dévote de bonne fabrique. ${ }^{39}$

La désacralisation subversive de l'odeur de sainteté, envisagée ici comme symptôme de névrose, et son association avec des causes érotiques, voire avec une pathologie mentale, remettent en cause la pertinence de convoquer les vies de saints pour l'édification des jeunes filles.

Ceci dit, la question du parfum ne peut être détachée de celle de ses origines religieuses. $\mathrm{Au} \mathrm{XIX}^{\mathrm{e}}$ siècle, l'encens continue de faire partie intégrante de la liturgie. Ainsi, dans Marguerite à vingt ans, Victorine Monniot décrit la visite d'un temple protestant en ces termes: «Ici, pas d'autel ralliant tous les cœurs dans l'adoration, le recueillement et la prière... Pas de tabernacle, foyer brûlant d'amour, d'où s'échappent les rayons enflammés qui ravissent l'âme jusque dans le sein de Dieu... [...] L'on n'y respire point ce parfum d'encens et de Divinité, qui transforme nos temples en vestibules du ciel ${ }^{40}$. Considéré, depuis l'Antiquité, comme un moyen de communication avec les Dieux, le parfum, dans le contexte religieux, est souvent brûlé, ce qui favorise son ascension vers le divin. Or, à l'ère industrielle, la fumée est dotée de connotations fort négatives. Au XIX ${ }^{\mathrm{e}}$ siècle, les parfums sont généralement liquides et dilués comme en témoignent les eaux de Cologne et eaux de toilette; ils s'inscrivent dans des pratiques de toilette utilisant abondamment l'eau. L'usage de parfums diffusés sous forme de fumée, tel l'encens des églises, est alors perçu comme un vestige archaïque ${ }^{41}$ qui affecte trop fortement les sens. Les représentations visuelles de l'époque témoignent de ces conceptions en faisant du brûleparfum un objet caractéristique des décors antiques et orientalistes (fig. 2).

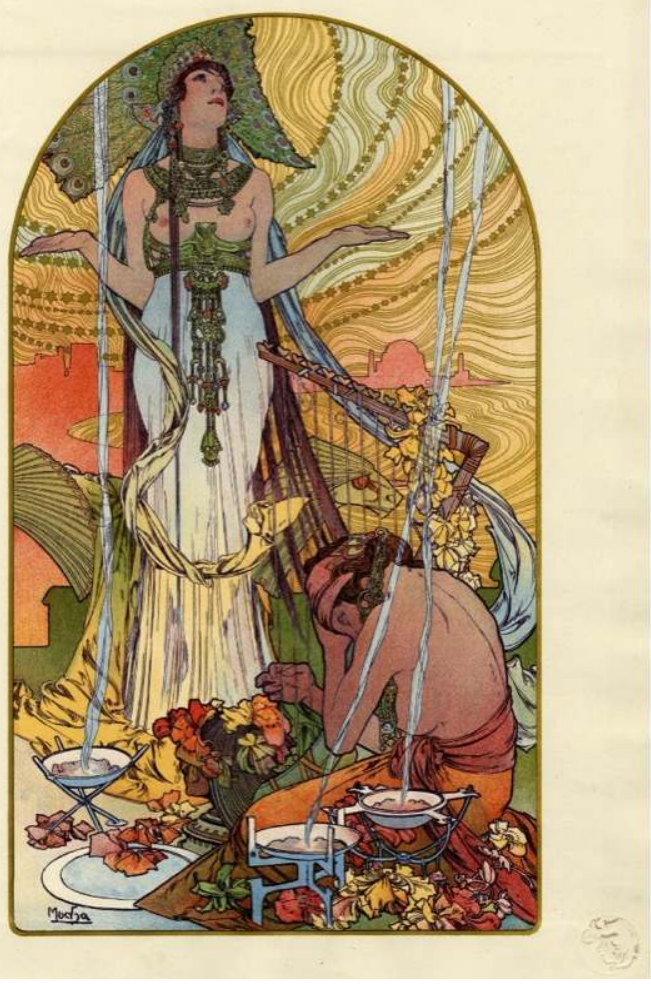

Fig. 2

Mucha, Incantation - L'estampe moderne, 1897, lithographie couleur, 37×21cm British Museum 
En règle générale, l'encens liturgique n'échappe pas à la condamnation des parfums par les médecins qui décèlent dans l'accoutumance à cette odeur forte et capiteuse, entretenue par la fréquentation des églises, le risque d'une exacerbation de la sensualité. Un tel parfum ne saurait être toléré par les femmes distinguées qui font de leur sensibilité olfactive une vertu morale: "[M]oi qui ne suis pas une femme à vapeurs, observe Madame Laurin, l'un des personnages de l'ouvrage de Madame Dumoulin consacré aux cinq sens, « je me trouve péniblement affectée par l'encens, par le patchouli, par le musc, que bon nombre de gens considèrent comme des parfums agréables $»^{42}$. Auguste Galopin va plus loin avec la mise en garde suivante: «Les jeunes personnes et les femmes, qui s'oublient dans l'extase amoureuse, ne tarderont pas à se narcotiser d'encens et d'autres pieuses odeurs, si rien ne vient détourner leur esprit et remuer leur cœur. $»^{43} L^{\prime}$ 'encens se trouve ainsi doté de tout le potentiel érotique que l'on prêtait au parfum. Alors que le discours religieux et le discours médical du Second Empire tendaient à opposer aux produits de parfumeries l'odeur abstraite et symbolique des vertus, la fumée d'encens introduit des ambiguités qui compromettent son rôle dans l'éducation des jeunes filles ${ }^{44}$. L'usage de l'encens donne lieu, comme l'absence de bain, à une dissension entre le discours religieux et le discours médical en matière d'éducation des jeunes filles : pour les uns, il élève l'âme ; pour les autres, il trouble l'esprit.

Les odeurs et les parfums, qu'ils émanent des jeunes filles ou qu'ils soient respirés par elles, sont ainsi placés au cœur des différents discours, médical, pédagogique et religieux, qui leur sont consacrés ou destinés. Au XIX ${ }^{e}$ siècle, la maîtrise des odeurs, incarnée par l'hygiène et la parfumerie, est associée à des enjeux sociaux majeurs sur le rôle réservé aux jeunes filles. Parallèlement, le rôle de l'olfaction dans l'éducation religieuse se révèle une source de controverses. L'interdiction faite aux jeunes filles d'utiliser du parfum alors qu'on les encourage par ailleurs à assister à des messes où l'on brûle de l'encens, n'est pas le moindre paradoxe d'une éducation dont nombre de médecins soulignent les effets pervers, tant d'un point de vue hygiénique que moral. Les promoteurs de l'hygiène et de la parfumerie comme facteurs de progrès dénoncent en effet l'archaïsme de l'encens et des pratiques de toilette que l'on peut s'étonner, avec Odile Arnold, de voir perdurer jusque dans les années $1950^{45}$. L'olfaction se trouve ainsi placée non seulement à l'articulation du matériel et du spirituel, du corps et de l'âme, mais aussi au carrefour de conceptions du corps divergentes.

\section{NOTES}

1. Alfred de Musset, "Rolla ", La Revue des deux mondes, t. III, août 1833, p. 371. Je remercie chaleureusement Eugénie Briot, Isabelle Saint-Martin, Mathilde Leichle et Émilie Sermadiras pour leurs précieux conseils en ce qui concerne les sources premières et secondes.

2. On a longtemps pensé, par exemple, que les odeurs se déplaçaient comme les ondes sonores, comme on peut le lire encore à la fin du siècle : « De nos jours encore cette hypothèse [la théorie dynamique], qui cherche l'origine de ces impressions dans des vibrations déterminées par les molécules odorantes et transportées à notre organe olfactif par l'atmosphère ou un autre milieu,

Arts et Savoirs, $11 \mid 2019$ 
ne manque pas de partisans » (Frantz Dussaud et M. Rubbrecht, Action physiologique des parfums, Paris, Georges Carré et C. Naud, 1897, p. 4).

3. Voir à ce sujet l'ouvrage de référence d'Alain Corbin, Le Miasme et la jonquille: L'odorat et l'imaginaire social XVIII ${ }^{e}$-XIX ${ }^{e}$ siècles, Paris, Aubier-Montaigne, 1982.

4. Éléonore Reverzy, «Parfums de (petites) femmes : pour une lecture olfactive », Littérature, $\mathrm{n}$ - 185, 2017, p. 55-67. Voir aussi Sophie-Valentine Borloz, «Les Femmes qui se parfument doivent être admirées de loin ": les odeurs féminines dans Nana de Zola, Notre cour de Maupassant et L'Ève future de Villier de l'Isle-Adam, Lausanne, Archipel, 2015.

5. Érika Wicky, «Ce que sentent les jeunes filles », Romantisme, n 165, 2015, p. 43-53. Nombre de médecins articulent le passage de l'enfance à l'âge adulte au rapport entre le végétal et l'animal. Voir à ce sujet Alain Corbin, L'Harmonie des plaisirs: les manières de jouir du siècle des Lumières à l'avènement de la sexologie, Paris, Perrin, 2007.

6. Rebecca Rogers, From the Salon to the Schoolroom: Educating Bourgeois Girls in Nineteenth-Century France, University Park, Pennsylvania State University Press, 2005, p.151. Voir également Gabrielle Houbre, "Demoiselles catholiques et misses protestantes: deux modèles éducatifs antagonistes au XIX ${ }^{\mathrm{e}}$ siècle », Bulletin de la Société de l'histoire du protestantisme français, t. 146, 2000, p. 49-68.

7. Eugénie Briot, La Fabrique des parfums: naissance d'une industrie de luxe, Paris, Éditions Vendémiaire, 2015.

8. Lettre de George Sand à son frère Hippolyte, citée dans René Doumic, George Sand: dix conférences sur sa vie et son œuvre, Paris, Perrin, 1909, p. 44. Voir également Isabelle Bricard, Saintes ou pouliches : l'éducation des jeunes filles au XIX siècle, Paris, Albin Michel, 1985.

9. Germain Sicard, "L'Offensive de la laïcisation de 1870-1871 », Revue d'histoire de l'Église de France, t. LXXXI, n² 206, 1995, p. 35-46.

10. Michel Launey, L'Église et l'école en France : XIX ${ }^{e}-X X^{e}$ siècles, Paris, Desclée, 1988, p. 63.

11. George Sand, Histoire de ma vie, t. III, Saint-Cyr-sur-Loire, Pirot, 1994, p. 154.

12. Le Livre des jeunes filles: conseils aux jeunes personnes par une religieuse de la nativité, Lyon / Paris, Librairie Girard \& Josserand. 1868, p. 216. Voir aussi à ce sujet Odile Arnold, Le Corps et l'âme : la vie des religieuses au XIX $x^{e}$ siècle, Paris, Seuil, 1984, p. 72.

13. Georges Vigarello, Le Propre et le sale: l'hygiène du corps depuis le Moyen Âge, Paris, Seuil, 1985. La pratique du bain devient d'autant plus importante que, tout d'abord déconseillée, elle a été fortement recommandée, à partir des années 1830, à toutes les catégories sociales, et ce, en dépit des réticences. Selon Georges Vigarello, l'engouement pour la pratique du bain à partir du milieu du siècle s'explique par l'évolution du discours médical ainsi que par les avancées dans la distribution de l'eau passée, selon l'historien, de 7,5 litres par jour et par personne en 1840 à 114 litres en 1873. Elle conduira à l'installation de salles de bains tout d'abord chez les personnes les plus aisées, puis dans les immeubles de rapport au cours des années 1880 (Georges Vigarello, « Hygiène du corps et travail des apparences », in Histoire du corps, t. 2, Alain Corbin, Jean-Jacques Courtine, Georges Vigarello (dir.), Paris, Seuil, 2005, p. 299-312).

14. Judith Gautier, Le Collier des jours : souvenirs de ma vie, Paris, F. Juven, 1904, p. 161. L'auteure rapporte également, plus loin, un mot de son père qui disait ne pouvoir comprendre les religieux qui « se réunissent pour puer de compagnie, en l'honneur d'un Dieu qui a créé dix mille espèces de parfums » (p. 155).

15. Jean-Baptiste Venot, Rapprochements statistiques entre les deux prostitutions, inscrite et clandestine, au point de vue de la syphilis : hygiène, Bordeaux, De G. Gounouilhou, 1857, p. 15.

16. Augustin Galopin, Le Parfum de la femme et le sens olfactif dans l'amour, étude psycho-physiologique, Paris, E. Dentu, 1886, p. 197.

17. Adrien Coriveaud, Hygiène de la jeune fille, Paris, Baillière, 1882, p. 87.

18. «Il ne faut laisser dans une chambre à coucher, ni fleurs trop odorantes, comme la rose, l'œillet ou la tubéreuse, ni fruits très-parfumés, comme les abricots, les melons et les pommes 
mûres, ni aucune espèce de parfum ", souligne le Docteur Louis Cyprien Descieux: «il en pourrait résulter pendant le sommeil, non pas seulement des maux de tête et des vertiges, mais une véritable asphyxie " (Manuel d'hygiène à l'usage des élèves des écoles normales primaires, des écoles spéciales, des lycées, collèges et séminaires, Paris, P. Dupont, 1867, p. 141).

19. Émile Zola, La Faute de l'Abbé Mouret, Paris, G. Charpentier, 1875, p. 408-415.

20. "Elle vivait donc au milieu des "extraits triples d'odeurs" à baptême anglais : Kiss me quick, Lily of the Valley, - New Moon Hay, - Spring Flowers, - West-End, - White-Rose, - White-Lilac, Ylang-Ylang. Et tous les "bouquets", tour à tour, Chérie en portait, chez elle ou dans le monde, les terribles aromes, - tous les bouquets, depuis le "bouquet de l'Impératrice Eugénie" jusqu'au "bouquet des Baisers dérobés", bouquets qui toujours enveloppaient la jeune fille du mélange flottant des esprits de tubéreuse, de fleurs d'oranger, de jasmin, de vétyver, d'opoponax, de violette, de fèves de Tonka, d'ambre gris, de santal, de bergamote, de néroli, de romarin, de benjoin, de verveine, de patchouli » (Edmond de Goncourt, Chérie, Paris, G. Charpentier et Cie, 1884, p. 223). Voir à ce sujet Andrea Oberhuber et Érika Wicky, «Du mauvais usage des parfums : Chérie empoisonnée par le musc et l'héliotrope ", Cahiers Edmond et Jules de Goncourt, n²3, 2016, p. $130-141$.

21. Jean-Baptiste Fonssagrives, Entretiens familiers sur l'hygiène ( $2{ }^{\mathrm{e}}$ édition) Paris, Hachette / Masson et fils, 1869, p. 295. On notera que l'hygiène est souvent invoquée dans une perspective religieuse, à titre, dès lors, d'hygiène morale. Voir, par exemple, Max Simon, Hygiène du corps et de l'âme, ou Conseils sur la direction physique et morale de la vie adressés aux ouvriers des villes et des campagnes, Paris, J. B. Baillière, 1853.

22. Alain Corbin, Le Miasme et la jonquille, op. cit., p. 80-81.

23. Les considérations sociales sont également parfois présentes dans le discours religieux. Un directeur de conscience écrit ainsi à une jeune fille: «Ma préoccupation à moi est délicate à exprimer; je suis trop votre père pour ne pas l'oser ; comprenez-le donc par ceci : ces parfums violents sont le partage à peu près exclusif de femmes que vous ne devez pas copier. Je crois que c'est en dire assez... » (Léopold Beaudenom, Lettres de direction, vers 1900, cité par Caroline Muller dans son carnet de recherche Acquis de conscience : Histoire(s) de direction de conscience au XIX ${ }^{e}$ siècle, https://consciences.hypotheses.org/151, consulté le 30 juin 2017).

24. Ce type d'imagerie fait partie de la culture visuelle des jeunes filles. Voir à ce sujet Isabelle Saint-Martin, Voir, savoir, croire: Catéchisme et pédagogie par l'image au XIX siècle, Paris, Honoré Champion, 2003. Sur le rôle de l'image dans l'éducation religieuse au XIX ${ }^{\mathrm{e}}$ siècle, voir également Isabelle Saint-Martin, Art chrétien / art sacré : Regards du catholicisme sur l'art. France, XIX ${ }^{e}-\mathrm{XX}{ }^{e}$ siècles, Rennes, Presses universitaires de Rennes, 2014, p. 91.

25. Marie Oberthal, De l'éducation morale de la femme : conseils à une jeune fille, Saint-Malo, E Renaud, 1865. p. 18.

26. «Souvenez-vous, du reste, que la propreté extérieure est regardée par les saints comme l'indice de la netteté et de la pureté de l'âme » (Le Livre des jeunes filles : conseils aux jeunes personnes par une religieuse de la nativité, Lyon / Paris, Librairie Girard \& Josserand. 1868, p. 215). Cette équivalence s'inscrit dans un imaginaire religieux très ancien, dont Jean-Pierre Albert a étudié les manifestations au Moyen Âge (Jean-Pierre Albert, Odeurs de sainteté: La mythologie chrétienne des aromates, Paris, Éditions de l'EHESS, 1996). Laurence Pfeffer rappelle ainsi que le bien et le mal, Dieu et le diable s'affrontent sur le terrain des odeurs (Laurence Pfeffer, "Odeur", Dictionnaire du corps, Michela Marzano (dir.), Paris, PUF, 2007, p. 670).

27. Émile Littré, Dictionnaire de la langue française, t. II, Paris, Hachette, 1874, p. 1493.

28. «Telles que ces fleurs modestes toutes parfumées qui embaument nos prairies et dont la culture s'est plu à doubler les feuilles, à agrandir les pétales et à réchauffer les nuances, - on reconnait bien encore la petite fleur des champs", s'exclame une mère dans un "Appel aux femmes russes », « mais le parfum en est perdu ! - les femmes russes de notre temps apprennent tout, parlent de tout, fouillent dans les secrets de l'anatomie et les mystères de la physique ; mais 
aux ronces de la science, elles ont mis en lambeaux leur innocence et leur pudeur, ce parfum délicat de la femme vertueuse » (Une mère, "Un appel aux femmes russes », La Femme, 1er août 1879, p. 116). De même, l'inspecteur de l'Académie de Lyon fait l'observation suivante dans un rapport du 3 mai 1868 : "Le savoir des femmes est comme les fleurs; il ne doit pas être exposé à un soleil trop ardent ; il doit être le parfum du foyer domestique » (cité par Rebecca Roger in «Le professeur a-t-il un sexe? Les débats autour de la présence d'hommes dans l'enseignement secondaire féminin, 1840-1880 ", Clio, $\mathrm{n}^{\circ} 4,1996$, p. 9).

29. Les plaisirs des sens sont généralement prohibés. Par exemple, dans Le Journal de Marguerite, best consacré à l'édification des jeunes filles sous le Second Empire, l'odeur n'est évoquée que pour signifier le dégoût (Victorine Monniot, Le Journal de Marguerite, Paris, Bourguet-Calas, 1878). Comme l'explique Alain Corbin : «L'essentiel réside dans la maîtrise des cinq sens. [...] Elle [la religieuse] ne saurait se délecter des bonnes odeurs. En revanche, elle peut rechercher les puanteurs, surtout lorsque celles-ci suggèrent l'accomplissement d'un geste charitable » (Alain Corbin, «L'emprise de la religion », Histoire du corps, t. 2, op. cit., p. 73).

30. Victorine Monniot évoque ainsi «les pauvres [qui] font monter vers le Ciel en parfum d'agréable odeur les mérites de leur résignation, de leur humilité, de leur reconnaissance » ( $a$ petite concierge, cité par Madeleine Lassère in Victorine Monniot ou l'éducation des jeunes filles au XIX siècle, Paris, L'Harmattan, 1999, p. 122).

31. En la matière, les fleurs des champs s'opposent aux fleurs exotiques, qui, alors perçues comme rares et sophistiquées, s'inscrivaient dans un tout autre registre que celui des jeunes filles. Artificiellement cultivées dans des serres contrairement aux fleurs sauvages, elles sont souvent dotées des connotations sensuelles de l'orientalisme. Voir Arnaud Verret, « La serre dans le roman zolien: un exemple d'insularisation de l'érotisme fin-de-siècle », in L'Éros insulaire, Alexandra W. Albertini et Jacques Isolery dir., Paris, Petra, 2016, p. 109-123.

32. Clarisse Juranville, Le Savoir-faire et le savoir-vivre dans les diverses circonstances de la vie. Guide pratique de la vie usuelle à l'usage des jeunes filles, Paris, Boyer, 1879, p. 29.

33. Jean-Baptiste Capefigue, La vénérable mère sainte Françoise de Chantal : fondatrice des Dames de la Visitation (visitandines), Paris, Amyot, 1865, p. 86.

34. Jean-Nicolas Bouilly, Conseils à ma fille, Paris, Rosa, 1813, p. 352-353.

35. «Le parfum se conserve suave et odorant dans l'urne qui le renferme, il perd son odeur si le cristal se brise et le laisse s'épancher au dehors ", observe le Révérend Père Trophime Roucau en citant les propos d'une jeune fille : « la fleur se garde fraîche, veloutée à l'abri de la haie épineuse qui la protège; elle se fane, touchée par la main de ceux qui l'admirent» (Chemin d'ombre, causeries : aux jeunes filles, Paris, P. Lethielleux, 1905, p. 190). Inversement, l'apparition de messes tardives permettant aux mondaines de se lever ou de se coucher plus tard a été critiquée par l'évocation du patchouli à l'odeur forte et tenace. Voir à ce sujet Clara Sadoun-Édouard, «Des confessionnaux embaumant le patchouli: Catholicisme et mondanité dans La Vie parisienne (1863-1900) ", Archives de sciences sociales des religions, $n^{\circ} 165,2014$, p. 143-161.

36. Ainsi, une forme de mémoire olfactive semble en mesure de rappeler le souvenir des pratiques religieuses au milieu des obligations mondaines : «... j'entends sonner l'heure de la prière, et je me souviens de nos sacrifices, de nos chants pieux, des fleurs et de l'encens que nous offrons à notre Dieu, et je retrouve au fond de mon cœur, comme un reste de parfum, comme un reflet de bonheur » (Madame Augustine de G.R., L'Éducation des jeunes filles sous l'influence de la foi, Paris, Charles Douniol, 1862, p. 340).

37. Dès les années 1860, par exemple, les progrès de la chimie permettent de produire à moindre coût des parfums artificiels. Voir, à ce sujet, Eugénie Briot, "Le marché français des parfums artificiels au XIX ${ }^{\mathrm{e}}$ siècle, entre défiance et démocratisation ", in L'Industrie chimique en question, Paris, Picard, 2010, p. 137-147.

38. Voir Constance Classen, "The breath of God: Sacred histories of scent", in The Color of Angels: Cosmology, Gender, and the Aesthetic Imagination, Londres/New York, Routledge, 1998, p. 36-60. Voir 
également Jean-Louis Benoît, "Autour de l'odeur de sainteté: les parfums dans le monde chrétien ", Iris, n 33, 2012, p. 55-89. Huysmans, pour sa part, introduit l'olfaction dans son récit de la vie de Sainte Lydwine de Shiedam, ouvrage qui ne s'adresse pas particulièrement aux jeunes filles, mais qui rend compte de l'imaginaire religieux fin-de-siècle : « En un constant miracle, il fit de ces blessures des cassolettes de parfums; les emplâtres que l'on enlevait, pullulant de vermine, embaumaient ; le pus sentait bon, les vomissements effluaient de délicats aromes ; et de ce corps en charpie qu'il dispensait de ses tristes exigences qui rendent les pauvres alités si honteux, il voulût qu'il émanât toujours un relent exquis de coques et d'épices du Levant, une fragrance à la fois énergique et douillette, quelque chose comme un fumet bien biblique de cinnamome, et bien hollandais, de cannelle » (Joris-Karl Huysmans, Sainte Lydwine de Shiedam, Paris, Plon, 1912 [1901], p. 89).

39. Augustin Galopin, op. cit., p. 201-202.

40. Victorine Monniot, Marguerite à vingt ans. Suite et fin du journal de Marguerite, Paris, BourguetCalas, 1879 , p. 78-79.

41. On pouvait déjà lire en 1850 que « la mode de brûler des parfums dans les boudoirs » était une manière de « complètement revenir au siècle de Louis XV » (La Mode, janvier 1850).

42. Mme Gustave Dumoulin, Les cinq sens, Paris, Hachette, 1881, p. 80.

43. Augustin Galopin, op. cit., p. 199. La critique du caractère sensuel de la dévotion est également très présente chez les auteurs naturalistes. Ce phénomène a été analysé par Éléonore Reverzy dans son article «La passion religieuse: les Goncourt, Zola et la question anticléricale ", Romantisme, $\mathrm{n}^{\circ}$ 107, 2000, p. 59-70 ainsi que dans son ouvrage La Mort d'Éros: La Mésalliance dans le roman du second XIX ${ }^{e}$ siècle, Paris, SEDES, 1997, p. 143-170.

44. Les plaisirs sensuels rencontrés à l'église sont évoqués dans de nombreux romans tel Germinie Lacerteux des frères Goncourt [1865]. Dans La Fille de Nana d'Alfred Sirven et Henri Leverdier, Andrée, fille de Nana, « respirait, avec une sorte de sensualisme éthéré, les parfums de l'encens montant vers les voûtes, dans le rayonnement des vitraux, et enveloppant de spirales bleues la grande croix d'or érigée sur le maître-autel » (Paris, E. Dentu, 1881, p. 37).

45. Odile Arnold, op. cit., p. 73.

\section{RÉSUMÉS}

Émanations invisibles et impalpables, les odeurs sont au cœur des liens étroitement noués au XIX siècle entre hygiène corporelle et moralité. Comme l'a bien montré Alain Corbin, les conceptions de l'olfaction renforcent l'articulation entre le corps et l'esprit, instaurant entre senteurs et vertus un rapport de causalité. Ainsi, l'odeur sous-tend souvent la dénonciation et l'opprobre des prostituées, mais elle joue aussi un rôle essentiel dans l'éducation des jeunes filles à laquelle est apporté, durant la seconde moitié du XIX ${ }^{\mathrm{e}}$ siècle, un soin tout particulier.

As invisible and impalpable emanations, odors were central to the close ties that the nineteenth century created between bodily hygiene and morality. As Alain Corbin has shown, notions of olfaction reinforced the articulation between the body and mind, establishing a relation of causality between scents and virtues. Consequently, odor often underpinned the denunciation of prostitutes and the shame cast upon them. At the same time, odor also played an essential role in the education of young girls, which was the object of a very particular attention during the second half of the nineteenth century. 
INDEX

Mots-clés : éducation, XIXe siècle, jeunes filles, odeurs, parfums

Keywords : éducation, 19th centurie, young girls, odors, perfumes

\section{AUTEUR}

\section{ÉRIKA WICKY}

Collegium de Lyon 